\title{
Provision of Recommended Chronic Pain Assessment and Management in Primary Care: Does Patient-Centered Medical Home (PCMH) Recognition Make a Difference?
}

\author{
Nancy Elder, MD, MSPH, Michelle Penm, B. Pharm (Hons), MBBS, MMed (Pain \\ Management), Harini Pallerla, MS, Mary Beth Vonder Meulen, RN, \\ Amy Diane Short, MHSA, Tiffiny Diers, MD, Ryan Joseph Imboff, BS, Brendan Wilson, \\ and Jill Martin Boone, PharmD
}

Background: Chronic pain (CP) care in the patient-centered medical home (PCMH) model has not been well studied. We assessed whether PCMH recognition is associated with increased provision of key practice recommendations for $\mathrm{CP}$ assessment and management.

Methods: Chart reviews were completed for 12 primary care practices affiliated with one health system in the Cincinnati Area Research and Improvement Group (CARInG). Recommendations were abstracted and compared based on PCMH status: 3 practices had received prior PCMH level 3 recognition, 5 were in an ongoing process of applying, and 4 had no recognition and were not applying.

Results: A total of 485 charts were reviewed from 65 PCPs. Eight of 10 key recommendations were documented more often in the prior and ongoing PCMH cohorts, including assessing pain severity, function, psychosocial distress, and substance abuse, and using structured instruments for these assessments. There were fewer differences between the cohorts in the management of chronic opioids, with only the ongoing PCMH cohort having higher documentation for 5 of the 7 recommendations, including performing urine drug screens and using a structured instrument to assess for misuse.

Conclusions: These findings support the usefulness of the PCMH model in managing patients with CP, but patient outcomes need to be addressed in future studies.(J Am Board Fam Med 2016;29:474-481.)

Keywords: Opioid Analgesics, Arthralgia, Chronic Disease, Chronic Pain, Documentation, Empathy, Humans, Internship and Residency, Musculoskeletal Pain, Patient-Centered Care, Primary Health Care, Substance-Related Disorders

Chronic pain (CP) is defined as pain that persists beyond 3 months, or pain that persists past the

This article was externally peer reviewed.

Submitted 26 January 2016; revised 13 April 2016; accepted 18 April 2016.

From the Department of Family and Community Medicine, University of Cincinnati, Cincinnati, OH (NE, HP, MBVM, $\mathrm{RJI})$; the University of Cincinnati, Cincinnati, $\mathrm{OH}$ (MP, BW); the Center for Improvement Science (ADS); Department of Internal Medicine, University of Cincinnati, Cincinnati, $\mathrm{OH}$ (TD); University of Cincinnati Center James L. Winkle College of Pharmacy, Cincinnati, OH (JMB).

Funding: Funding was provided by a Pfizer Independent Grant for Learning and Change.

Conflict of interest: none declared.

Corresponding author: Nancy Elder, MD, MSPH, Department of Family and Community Medicine, University of Cincinnati, PO Box 670582, Cincinnati, OH 45267 (E-mail: Nancy.Elder@uc.edu). expected healing time. ${ }^{1,2}$ The cost of CP to individuals and society is enormous, with tens of millions of adults suffering from CP, costing up to $\$ 635$ billion annually. ${ }^{3}$ Furthermore, the increasing use of prescription opioids for the management of $\mathrm{CP}$ has resulted in increases in opioid dependence and deaths caused by overdose. ${ }^{4}$

Primary care providers (PCPs) are often the first point of contact for patients with $\mathrm{CP}$ and are in an ideal place for the ongoing care and management of these patients. ${ }^{3}$ Currently, over half of patients with CP are cared for by PCPs. However, these providers find patients with $\mathrm{CP}$ a challenge to assess and manage. ${ }^{5}$ There is great variability in PCPs' adherence to pain documentation and opioid prescribing guidelines. ${ }^{6-8}$ Many PCPs are unaware 
of guidelines that are available to help them treat patients with CP; only $38 \%$ of PCPs surveyed were aware of at least 1 clinical practice guideline for CP. ${ }^{9}$ There is also minimal use of monitoring tools such as pain assessment scales, urine drug screens, and documentation of treatment plans, even in high-risk patients receiving opioid therapy. ${ }^{6,10-12}$

There is a need for better systems of care for patients with CP. Long waits to access pain management and lack of access to addiction medicine and integrative and mental health specialists are barriers in CP management. ${ }^{6,13}$ Reimbursement policies may limit patient-centered care, as PCPs have limited time for planning, performing comprehensive assessments, and coordinating care. ${ }^{3}$ Further, there may be little teamwork or few structured opportunities for communication between office staff and PCPs, resulting in important information divulged by patients to staff, which is then not relayed to PCPs. ${ }^{11}$

Recent health care reforms have focused on the patient-centered medical home $(\mathrm{PCMH})$ as a model to provide comprehensive primary care to patients. ${ }^{14}$ Although not a new concept (the American Academy of Pediatrics introduced the concept of a medical home in 1967), the current PCMH model incorporates aspects of the Chronic Care Model to facilitate partnership between patients and physicians. ${ }^{15}$ Key components of the PCMH include whole-person orientation, integrated and coordinated care, a personalized relationship with a physician, and continuous quality and safety improvements. ${ }^{16}$ Processes now exist for the recognition of practices as PCMHs, of which that of the National Committee for Quality Assurance (NCQA) has become the most prominent. ${ }^{17}$

The success of the PCMH model with other chronic diseases and its emphasis on comprehensive and team-based care suggest that it may provide an excellent structure for the treatment of CP. ${ }^{15,18,19} \mathrm{PCMH}$ innovations were shown to improve physician attitudes and satisfaction in treating patients with $\mathrm{CP}$ in a resident primary care practice, ${ }^{20}$ but changes in the provision of $\mathrm{CP}$ care are not known. We do not know whether practices that undergo PCMH transformation and obtain NCQA PCMH recognition actually provide better care for patients with CP. As part of a larger study of $\mathrm{CP}$ in primary care, we compared the documentation of key guideline criteria for primary care assessment and management of patients with CP by
PCMH recognition status in primary care offices of the Cincinnati Area Research and Improvement Group (CARInG) practice-based research network (PBRN).

\section{Methods \\ Participants}

All primary care (family medicine $[\mathrm{FM}]$, general internal medicine [GIM], and general internal medicine/pediatrics $[\mathrm{M} / \mathrm{P}]$ ) practices affiliated with the University of Cincinnati (UC Health) in Cincinnati, Ohio, were invited to participate in a study of CP care, and 12 of 14 practices agreed. These practices are a subset of the CARInG PBRN and were selected because they all used the same electronic health record (EHR; EPIC) and the same institutional review board (University of Cincinnati), which approved this study. As part of a larger study to improve CP care in primary care, a natural experiment occurred during the collection of retrospective baseline data in which the 12 practices were in 3 different $\mathrm{PCMH}$ recognition status subsets. Three practices (1 FM, 2 GIM) had achieved PCMH level 3 recognition before data collection, 5 practices (3 FM, 1 GIM, $1 \mathrm{M} / \mathrm{P}$ ) that were in an ongoing application process (for PCMH level 3), and 4 practices ( $1 \mathrm{FM}, 2$ GIM, $1 \mathrm{M} / \mathrm{P}$ ) with no $\mathrm{PCMH}$ recognition nor ongoing process during the year of data collection (July 1, 2012 through June 30, 2013). Details of the providers in these practices (from provider surveys collected in early 2013) are shown in Table 1; the prior PCMH group was the only group that included a residency practice (GIM).

\section{Data Collection}

We reviewed the charts of a sample of patients who received care for $\mathrm{CP}$ from providers in each practice. We developed a set of 30 common diagnosis codes for CP problems, including CP syndrome, low-back pain, joint (knee, hip, shoulder, etc.) pain, neuropathy, and fibromyalgia. Patients with at least 2 visits with these codes between July 2, 2012, and June 30, 2013, were generated from the patient database, and 6 to 15 patients per provider were randomly chosen for review. Charts were audited by a trained research nurse, and after confirmation that the patient had CP (pain for $>3$ months), all visits during the 12 -month period were reviewed and data were abstracted from visit notes, problem 
Table 1. Provider Demographics (Self-reported by Practices)

\begin{tabular}{lccc}
\hline Demographics & Prior PCMH & Ongoing PCMH & No PCMH \\
\hline Providers, n & 31 & 18 & 16 \\
Currently a resident* & 58.1 & 0 & 0 \\
Family medicine physician & 9.7 & 35.3 & 25 \\
Internal medicine physician & 77.4 & 11.8 & 68.8 \\
Internal medicine/pediatric physician & 12.9 & 29.4 & 6.3 \\
Nurse practitioner/physician assistant & 0 & 23.5 & 0 \\
Mean age (years)* & 34 & 45 & 37 \\
Female sex & 33 & 67 & 50 \\
Race/ethnicity & & & 68.8 \\
$\quad$ White & 66.7 & 18.8 & 67.5 \\
African American & 6.7 & 12.5 & 6.3 \\
Asian American & 26.7 & 0 & 6.3 \\
Hispanic & 6.5 & & 6.7 \\
\hline
\end{tabular}

Data are percentages unless otherwise indicated.

*Statistically significant between the 3 cohorts $(P<.001)$.

$\mathrm{PCMH}$, patient-centered medical home.

lists, medication lists, laboratory results, referrals, and consultant notes. Approximately 10\% of charts were also reviewed by the project's principal investigator (NE) to ensure the quality and accuracy of the data collection. In addition to information about types of CP documented anywhere in the chart (eg, musculoskeletal [back, joint], neuropathy, headache, pelvic), we noted assessment and management components; the documentation of key guidelines put forth by Corson et $\mathrm{al}^{21}$ were specifically noted: CP addressed, functional status addressed, pain severity measured quantitatively, psychosocial issues addressed, depression addressed, nonpharmacologic approach considered, substance use addressed, and, for those on opioids, side effects of opioids addressed. These key guidelines were selected because they had been used in primary care and they had fair to good quality ratings. ${ }^{21}$ Referrals to pain consultants (pain management, mental health, physical therapy, integrative medicine, and specific specialists [eg, orthopedics for joint pain, neurology for headaches]) were also documented. If a referral occurred before the 12-month review period but was referred to or discussed in the chart within this period, it was counted as a referral. If $>3$ prescriptions or notations of opioid use were documented in the past 6 months, the patient was considered to be on chronic opioids and further documentation of opioid monitoring and management were obtained.

\section{Data Analysis}

The presence of the key guideline criteria, monitoring of chronic opioid use, and pain consultant referrals in the charts were compared by when, and if, the practice has achieved NCQA recognition (before the data collection period, ongoing during the data collection period, or no PCMH recognition). The chart review data were analyzed using SPSS software (versions 22 and 23; IBM, Chicago, IL). Bivariate analyses were done to explore the relationships between all the dichotomized variables of the chart review with their site PCMH status. Statistical significance was calculated using the Fisher exact test $(P<.05)$.

\section{Results}

A total of 485 charts were reviewed from 65 clinicians at 12 practices. Demographics of the patients by PCMH recognition cohort are shown in Table 2. Musculoskeletal pain, including low-back and joint pain, was the most common type of pain (present in 93\% of the patients), followed by neuropathy (in 23\%) and chronic headaches (in 21\%) There were no significant differences in the types of pain diagnoses by PCMH cohort. Most patients had $\geq 2$ types of $\mathrm{CP}$, with significant differences between the cohorts ( $51 \%$ with $\geq 2$ types of $\mathrm{CP}$ in PCMHs, $68 \%$ in practices currently applying for recognition as a $\mathrm{PCMH}$, and $56 \%$ in practices not PCMHs $[P=.006])$. A majority of patients $(58 \%)$ 
Table 2. Patient Demographics from Chart Review of Patients with Chronic Pain

\begin{tabular}{lccc}
\hline & $\begin{array}{r}\text { Prior } \\
\text { PCMH }\end{array}$ & $\begin{array}{c}\text { Ongoing } \\
\text { PCMH }\end{array}$ & $\begin{array}{c}\text { No } \\
\text { PCMH }\end{array}$ \\
\hline Patients, n & 128 & 242 & 115 \\
Mean age (years)* & 52 & 56 & 59 \\
Female sex* $^{*}$ & 50.4 & 70.2 & 55.7 \\
Race/ethnicity* & & & \\
White & 70 & 50 & 88 \\
$\quad$ African American & 30 & 48 & 10 \\
Asian American/other & 0 & 2 & 2 \\
Hispanic & 1 & 1 & 2 \\
\hline
\end{tabular}

Data are percentages unless otherwise indicated.

*Statistically significant between the cohorts $(P<.001)$.

$\mathrm{PCMH}$, patient-centered medical home.

had also been prescribed chronic opioids, but there was no statistical difference in opioid use between the cohorts $(62 \%$ in $\mathrm{PCMHs}, 59 \%$ in practices currently applying for recognition as a PCMH, and $54 \%$ in practices not PCMHs $[P=$ not significant]).

\section{Key Guidelines}

Figure 1 documents the presence of key guidelines for the primary care of patients with $\mathrm{CP}$ in these patients' charts. Without exception, those practices with $\mathrm{PCMH}$ recognition or applying for recognition documented these recommendations more often, most at a statistically significant level. For many assessments, including pain severity and functional disability, the practices that were in the process of applying for $\mathrm{PCMH}$ recognition performed the best.

\section{Opioid Monitoring}

Over half the patients in each $\mathrm{PCMH}$ recognition cohort received chronic opioid prescriptions. Documentation rates of both practice recommendations and legal monitoring requirements for the state are found in Figure 2. In general there were fewer differences in the documentation of recommended opioid monitoring by PCMH status. The cohort currently in the process of obtaining

Figure 1. The provision of recommended chronic pain assessment and management by patient-centered medical home status (PCMH).

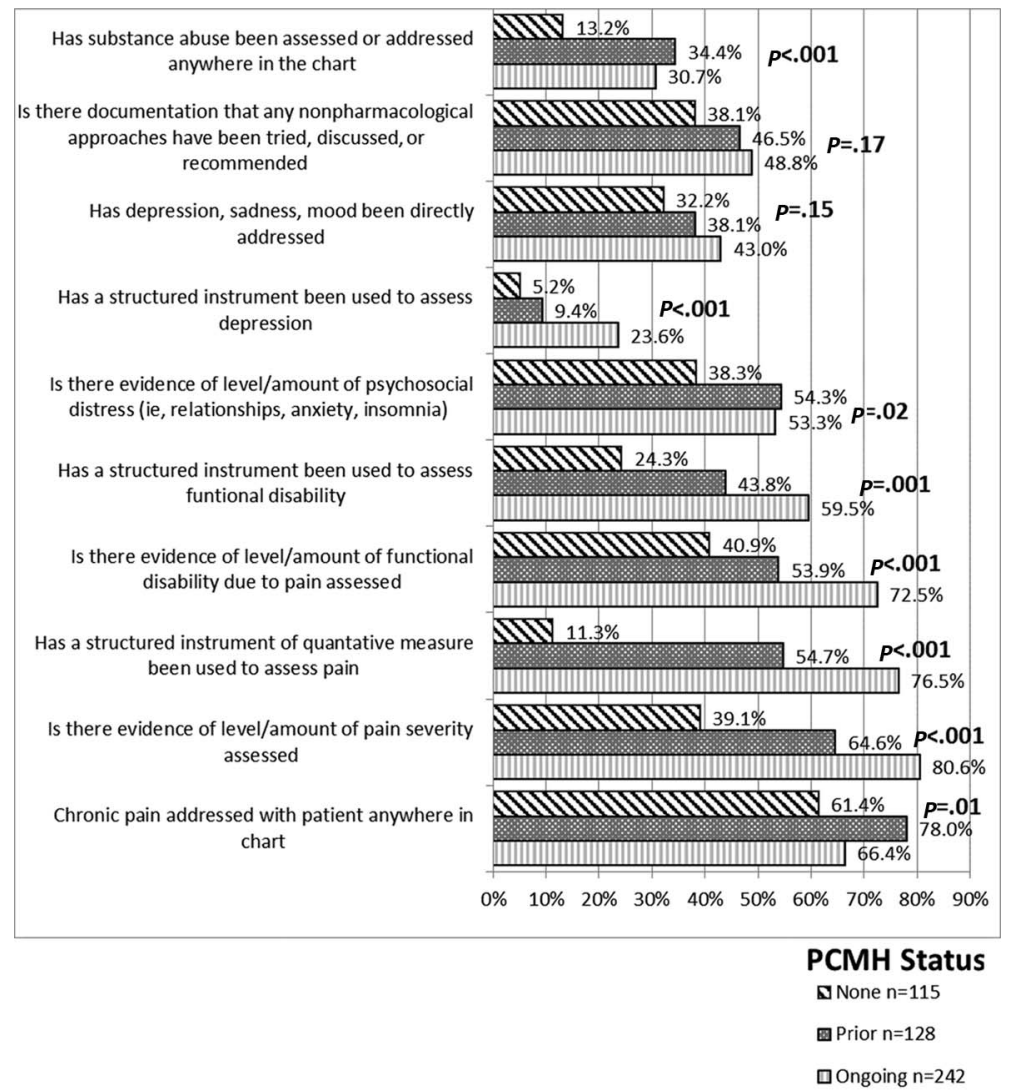


Figure 2. The provision of recommended chronic opioid assessment and management by patient-centered medical home status (PCMH).

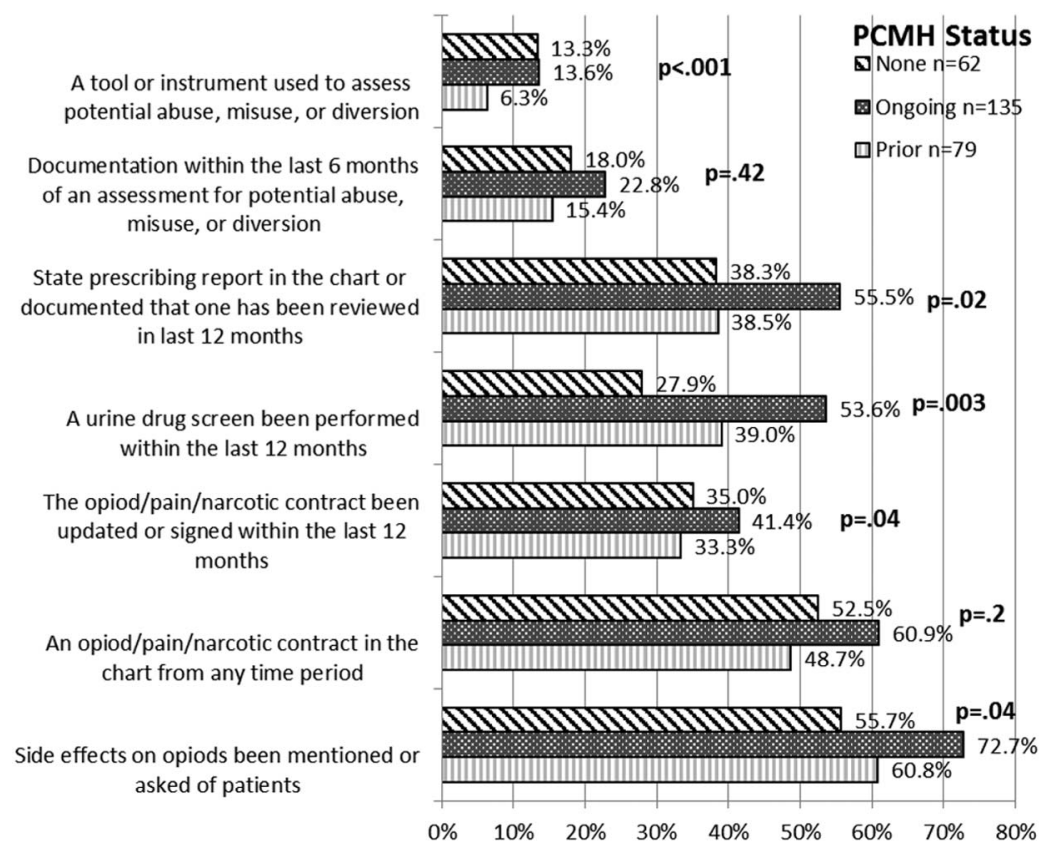

PCMH recognition achieved the highest levels for every item.

\section{Use of and Communication with Pain Consultants}

There were no statistically significant differences between the PCMH recognition cohorts in their use of a variety of pain consultants. Specialists specific to the pain problem (eg, orthopedic surgeons for joint pain) were the most commonly used; 57\% of patients had such a referral. Physical therapy was the second most common (35\% of patients), followed by pain management (32\%), mental/behavioral health $(11 \%)$, and integrative medicine $(3 \%)$.

\section{Discussion}

The number of primary care practices that have achieved NCQA PCMH recognition has increased nationally from 28 in 2008 to $>5500$ in $2013 .^{22}$ These practices commit to achieving a number of important elements, including population health management, care management, care coordination, performance measurement, and quality improvement. Research has shown that the PCMH model is associated with a number of positive outcomes, including better patient health outcomes for selected conditions, decreased health disparities, decreased health costs, and increased use of quality improvement (QI). ${ }^{23}$ No studies, however, have assessed the role of $\mathrm{PCMH}$ recognition on the provision of recommended care for CP. We found a higher rate of documentation of many key practice recommendations in practices that had previously achieved or were in the process of obtaining recognition compared with those who had no such recognition. We believe the focus of $\mathrm{PCMH}$ on quality and population management may explain these findings, although existing barriers to care coordination still limit high-quality interprofessional care for patients with CP.

Documentation of key measures of pain enables clinicians to better diagnose $\mathrm{CP}$, monitor treatment effectiveness, and observe for aberrant patient behavior. ${ }^{24}$ Part of the NCQA PCMH recognition process requires practices to adopt a system to improve the utilization of key practice guidelines. This requires an ability both to measure and improve practice and to identify populations of patients. ${ }^{25,26}$ Self-reported assessment of pain has been shown to be a reliable measure for pain and is a valuable resource to assess change and/or progress in treatment. ${ }^{27}$ However, the ability to identify the population of patients with $\mathrm{CP}$-which is crucial to providing quality care-is difficult and often lacking in practice. ${ }^{21,28}$ For example, many diagnostic codes do not differentiate between $\mathrm{CP}$ and acute pain, the 
underlying disease code (such as arthritis) does not necessarily address the presence of pain, and using the prescription of chronic opioids to define $\mathrm{CP}$ removes many patients with $\mathrm{CP}$ from the population. Complex algorithms have been developed to identify patients from existing EHR data ${ }^{29}$; however, simpler methods, such as adding a "CP syndrome" diagnosis to the problem list, are also effective, albeit rarely used. Using the EHR within the PCMH to create patient registries to identify patients with $\mathrm{CP}$ is also important for tracking patients who require follow-up such as opioid refills and urine drug screening. ${ }^{24}$

Interestingly, those practices submitting their PCMH application during the study year documented the highest rates of applying several key recommendations. This is especially true in the documentation of chronic opioid management, where the PCMH cohort performed similarly to the group with no PCMH recognition, but differences between all the cohorts were less significant. Actions by the State of Ohio during this time may have affected all practices: new guidelines for review of prescribing records were released in late 2011, and in May 2012 the Ohio Opiate Summit was held to establish statewide prescribing guidelines, which were released in May 2013. It may be that those practices currently in the PCMH transformation process were better situated to react to these changes. However, these findings raise the question of whether some of the benefits seen in studies of PCMH recognition may wane after a practice has achieved its initial recognition milestones. Further research into the effect of PCMH renewal is indicated.

Although the $\mathrm{PCMH}$ recognition process is associated with higher documentation of certain aspects of pain management, our findings show far from adequate provision of many key recommendations, such as using structured instruments to assess pain, function, depression, and opioid risk. Multiple barriers still exist in the implementation of optimal pain management. Physicians receive limited formal training and education in pain care, which affects patient assessment and management. ${ }^{3,8,30} \mathrm{CP}$ is often equated with chronic opioid use, leading to negative and ill-informed attitudes regarding patients with CP. Physicians cite past experiences with opioids and fears of regulatory scrutiny, of creating addicts, and of being "duped" by patients with $\mathrm{CP}$ as reasons for difficulties in caring for patients with CP. ${ }^{6,31}$ Patients with CP often suffer from significant psychiatric comorbidities such as depression, anxiety, and substance use disorders and find engaging with treatment difficult. ${ }^{6,32}$ Patients with CP report a lack of resources, including minimal support from family and friends and financial and transportation constraints, as further barriers to optimal pain management. ${ }^{32}$ Systemic barriers such as long waiting lists and lack of access to addiction, pain, and mental health specialists because insurance barriers and reimbursement policies also plague optimal CP management., ${ }^{6,13}$

There are several limitations to this study. For convenience, we studied only a subset of the CARInG PBRN, which uses a single EHR. Thus our study population is not necessarily representative of all practices in this region. Since we needed to manually review charts (because not all CP data are in searchable fields), we selected patients using diagnosis codes that were likely to yield a high percentage of "true-positive" patients with CP. There is a possibility that patients with less common pain complaints were excluded and may have been treated differently by their providers. The NCQA recognition process requires a set of administrative and process changes in a practice; there are many ways to achieve these changes, and practices with the same NCQA recognition vary greatly. We did not systematically evaluate the practices in any other way, but we did find that the 3 cohorts had some statistically significant differences in both the physician and patient makeups, such as a GIM residency in the $\mathrm{PCMH}$ cohort and patients who were slightly older in the no PCMH cohort. While there is potential that these differences, or other unmeasured differences, influenced the main findings, the overall number of physicians and practices is relatively small in this preliminary study, limiting the ability to model all demographic components. However, we believe the findings are important to advancing our understanding of the role of PCMH recognition in CP care and to suggest further studies.

\section{Conclusions}

The majority of patients with $\mathrm{CP}$ are cared for within primary care practices; however, the management of these patients is often considered onerous and burdensome for physicians and practice staff. $6,7,10,11,21,28,30$ The PCMH model aims to pro- 
vide comprehensive patient-centered care, and implementation of PCMHs in the primary care setting has resulted in improved adherence to guidelines and screening recommendations for common chronic disease states. ${ }^{23}$ Although the practices in our naturally occurring study were not matched for demographics or presence of a residency practice, our study is one of the first to examine the impact of $\mathrm{PCMH}$ recognition on the documentation of recommended care for patients with CP. We found that PCMH-recognized practices and practices in the process of obtaining PCMH recognition had higher rates of documenting key practice CP recommendations. These findings suggest the usefulness of the PCMH model in managing patients with CP. Further research is necessary to determine whether improved documentation of key practice guidelines produces sustainable and long-term improvements in patient outcomes.

\section{References}

1. Covington EC, Mathews M. Chronic nonmalignant pain. Lyndhurst, OH: Cleveland Clinic Foundation; 2010. Available from: http://www.clevelandclinicmeded.com/ medicalpubs/diseasemanagement/psychiatry-psychology/ chronic-nonmalignant-pain/Default.htm. Accessed September 23, 2015.

2. National Institutes of Health. Safely managing chronic pain. NIH MedlinePlus 2011;6:5-6.

3. Institute of Medicine. Relieving pain in America: a blueprint for transforming prevention, care, education, and research. Washington, DC: National Academies Press; 2011.

4. Centers for Disease Control and Prevention (CDC). Vital signs: overdoses of prescription opioid pain relievers-United States, 1999-2008. MMWR Morb Mortal Wkly Rep 2011;60:1487-92.

5. Breuer B, Cruciani R, Portenoy RK. Pain management by primary care physicians, pain physicians, chiropractors, and acupuncturists: a national survey. South Med J 2010;103:738-47.

6. Leverence RR, Williams RL, Potter $M$, et al. Chronic non-cancer pain: a siren for primary care-a report from the PRImary care MultiEthnic Network (PRIME Net). J Am Board Fam Med 2011; 24:551-61.

7. Anderson D, Wang S, Zlateva I. Comprehensive assessment of chronic pain management in primary care: a first phase of a quality improvement initiative at a multisite community health center. Qual Prim Care 2012;20:421-33.

8. Green CR, Wheeler JR, Marchant B, LaPorte F, Guerrero E. Analysis of the physician variable in pain management. Pain Med 2001;2:317-27.
9. Wolfert MZ, Gilson AM, Dahl JL, Cleary JF. Opioid analgesics for pain control: Wisconsin physicians' knowledge, beliefs, attitudes, and prescribing practices. Pain Med 2010;11:425-34.

10. Clark JD. Chronic pain prevalence and analgesic prescribing in a general medical population. J Pain Symptom Manage 2002;23:131-7.

11. Elder NC, Simmons T, Regan S, Gerrety E. Care for patients with chronic nonmalignant pain with and without chronic opioid prescriptions: a report from the Cincinnati Area Research Group (CARinG) Network. J Am Board Fam Med 2012; 25:652-60.

12. Starrels JL, Becker WC, Weiner MG, Li X, Heo M, Turner BJ. Low use of opioid risk reduction strategies in primary care even for high risk patients with chronic pain. J Gen Intern Med 2011;26:958-64.

13. Lynch ME, Campbell F, Clark AJ, et al. A systematic review of the effect of waiting for treatment for chronic pain. Pain 2008;136:97-116.

14. Patient Protection and Affordable Care Act. PL no. 111-148, H.R. 35902010, March 23, 2010.

15. Gabbay RA, Bailit MH, Mauger DT, Wagner EH, Siminerio L. Multipayer patient-centered medical home implementation guided by the chronic care model. Jt Comm J Qual Patient Saf 2011;37:265-73.

16. American Academy of Family Physicians (AAFP), American Academy of Pediatrics (AAP), American College of Physicians (ACP), American Osteopathic Association (AOA). Joint principles of the patientcentered medical home. Patient Centered Primary Care Collaborative. 2007. Available from: http:// www.aafp.org/dam/AAFP/documents/practice_management/pcmh/initiatives/PCMHJoint.pdf. Accessed June 1, 2016.

17. Weiss GG. PCMH: 6 ways to get started. Technology, care coordination, redefining staff roles keys to implementation success. Med Econ 2013; 90:14-7, 20.

18. Moureaux C, Perelman J, da Costa EM, et al. Impact of the medical home model on the quality of primary care: the Belgian experience. Med Care 2015;53: $396-400$.

19. Cheatle MD, Klocek JW, McLellan AT. Managing pain in high-risk patients within a patient-centered medical home. Transl Behav Med 2012;2:47-56.

20. Evans L, Whitham JA, Trotter DR, Filtz KR. An evaluation of family medicine residents' attitudes before and after a PCMH innovation for patients with chronic pain. Fam Med 2011;43:702-11.

21. Corson K, Doak MN, Denneson L, et al. Primary care clinician adherence to guidelines for the management of chronic musculoskeletal pain: results from the study of the effectiveness of a collaborative approach to pain. Pain Med 2011;12:1490-501.

22. National Committee for Quality Assurance. The power of partnering with NCQA: a force for positive change in health care. Investing in the future 
of quality health care for all Americans. 2013-2014 edition. Available from: http://www.ncqa.org/ Portals/0/Sponsor/NCQA\%20Case\%20Statement\% 202013-2014.pdf. Accessed May 20, 2016.

23. National Committee for Quality Assurance. Latest evidence: benefits of the patient-centered medical home. June 2015. Available from: https://www. ncqa.org/Portals/0/Programs/Recognition/NCQA\% 20PCMH\%20Evidence\%20Report, \%20June \% 202015.pdf. Accessed May 20, 2016.

24. Jackman RP, Purvis JM, Mallett BS. Chronic nonmalignant pain in primary care. Am Fam Physician 2008;78:1155-62.

25. National Committee for Quality Assurance. Patientcentered medical home (PCMH) 2014. Part 1: standards 1-3. Available from: https://www.ncqa.org/ Portals/0/Programs/Recognition/RPtraining/PCMH\% 202014\%20Intro.\%20Training\%20Slides\%20Part\% 201\%20-\%20Standards\%201-3\%20-\%2011.26.pdf. Accessed May 20, 2016.

26. National Committee for Quality Assurance. Patient-centered medical home (PCMH) 2014. Part 2: standards 4-6. Available from: https://www.ncqa. org/Portals/0/Programs/Recognition/RPtraining/
PCMH\% 202014\%20Intro.\%20Training\%20Slides\% 20Part\%202\%20-\%20Standards\%204-6\% 20-\% 2011.26.pdf. Accessed May 20, 2016.

27. Von Korff M, Jensen MP, Karoly P. Assessing global pain severity by self-report in clinical and health services research. Spine 2000;25:3140-51.

28. Clark LG, Upshur CC. Family medicine physicians' views of how to improve chronic pain management. J Am Board Fam Med 2007;20:479-82.

29. Tian TY, Zlateva I, Anderson DR. Using electronic health records data to identify patients with chronic pain in a primary care setting. J Am Med Inform Assoc 2013;20:e275-80.

30. Ponte CD, Johnson-Tribino J. Attitudes and knowledge about pain: an assessment of West Virginia family physicians. Fam Med 2005;37:477-80.

31. Olsen Y, Daumit GL. Opioid prescribing for chronic nonmalignant pain in primary care: challenges and solutions. Adv Psychosom Med 2004;25:138-50.

32. Bair MJ, Matthias MS, Nyland KA, et al. Barriers and facilitators to chronic pain self-management: a qualitative study of primary care patients with comorbid musculoskeletal pain and depression. Pain Med 2009;10:1280-90. 